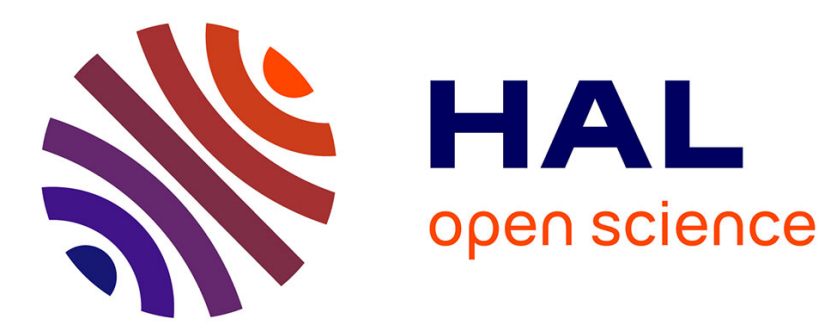

\title{
Controlled Mobility in Mobile Sensor Networks: Advantages, Issues and Challenges
}

\author{
Enrico Natalizio, Valeria Loscrì
}

\section{To cite this version:}

Enrico Natalizio, Valeria Loscrì. Controlled Mobility in Mobile Sensor Networks: Advantages, Issues and Challenges. Telecommunication Systems, 2013, 52 (4), pp.2411-2418. 10.1007/s11235-011-9561x . hal-00917519

\section{HAL Id: hal-00917519 https://hal.science/hal-00917519}

Submitted on 19 Dec 2013

HAL is a multi-disciplinary open access archive for the deposit and dissemination of scientific research documents, whether they are published or not. The documents may come from teaching and research institutions in France or abroad, or from public or private research centers.
L'archive ouverte pluridisciplinaire HAL, est destinée au dépôt et à la diffusion de documents scientifiques de niveau recherche, publiés ou non, émanant des établissements d'enseignement et de recherche français ou étrangers, des laboratoires publics ou privés. 


\title{
Controlled Mobility in Mobile Sensor
}

\section{Networks: Advantages, Issues and Challenges}

\author{
Enrico Natalizio* and Valeria Loscrí ${ }^{\dagger}$ \\ *INRIA Lille-Nord Europe, FRANCE. Email: enrico.natalizio@inria.fr \\ ${ }^{\dagger}$ DEIS - University of Calabria, Rende, ITALY. Email: vloscri@ deis.unical.it
}

\begin{abstract}
Recently, wireless self-organizing networks are attracting a lot of interest in the research community. Moreover, in the last decade many mobile devices have appeared in the market. Exploiting mobility in a wireless environment, instead of considering it as a kind of disturbance, is a fundamental concept that the research community is beginning to appreciate now. Of course, the advantages obtainable through the use of the mobility imply the knowledge of the different types of mobility and the way to include it in the management architecture of the wireless networks. In this work we claim that mobility and wireless sensor networks can be considered as two synergetic elements of the same reality. For this purpose, we sketch a macro-classification of the different objectives which can be pursued by controlled mobility. Moreover, we identify and highlight the interactions between this specific type of mobility and the layers of the control stack. Lastly, this paper reports a case study in which we show how controlled mobility can be exploited practically.
\end{abstract}

\section{Index Terms}

Controlled Mobility, Mobile Sensor Networks, Self-Organizing Networks

\section{INTRODUCTION}

Self-organization is a great concept for building scalable systems consisting of a large number of subsystems. Key factors in similar environments are coordination and collaboration of the subsystems for achieving a shared goal. In the last few years, the concept of self-organization has been applied to wireless networks. Also, in this context the self-organization concept can be summarized as the interaction of multiple components on a common global objective. This collaborative work may be realized through a central or distributed control and the primary objectives of similar networks are scalability, reliability and sustainability [19]. Moreover, with miniaturization of computing elements we have seen many mobile 
devices appear in the market that can collaborate in an ad hoc fashion without requiring any previous infrastructure control. This latter consideration allows us to consider the mobility as a fundamental aspect of the self-organizing networks. We can identify three macro-categories of mobility: random, predictable and controlled. In the first category, mobile devices are supposed to move according to a random mobility pattern. Many probabilistic models have been proposed in order to foresee devices' movements. Unfortunately random mobility represents more of a problem to solve than an advantage to exploit. A network access point mounted on a means of public transportation that moves with a periodic schedule represents a case of predictable mobility. A predictable schedule permits an easier, programmable accomplishment of some desired target, but mobility is not considered as a network primitive yet. Finally, controlled mobility generally consists of mobile devices introduced in the network and moving to specified destinations with defined mobility patterns for specific objectives. We can figure out many goals that could be achieved through controlled mobility, such as: coverage management [5], [6], [7], energy consumption reduction [1], [2], [3], [4], transport layer parameters' improvement [15], [17], [18]. This paper is specifically focused on controlled mobility, and its main contributions are:

1) We sketch out the research issues which arise when controlled mobility is integrated in the management architecture of a wireless sensor self-organizing network.

2) We highlight the advantages in the usage of controlled mobility by classifying research works which aim to optimize some network objective. The classification will take into account a series of aspects, such as the type of network, the objective of the research, the solution scheme (centralized/distributed), the type (programmed/adaptive) of controlled mobility and its penetration (one/some/all devices) in the network.

3) We propose some of the limitations that a self-organizing network design should take into account when controlled mobility is used.

4) We propose a case study in order to show advantages and detailed operations of controlled mobility, when it is considered as a network basic control factor.

The rest of the paper is organized as follows. In Section II, we analyze the relationships between controlled mobility and the communication protocols stack in order to show future challenges in the research. In Section III, we introduce controlled mobility and we explain its advantages and limitations. A case study is presented in Section IV and finally, the paper is concluded in Section V.

\section{Challenges LAYeR By LAYeR}

Fig. 1 (a) describes a self-organizing sensor network where, initially, nodes are monitoring their Voronoi cells. Reactively to specific events, as in Fig. 1 (b), nodes move in order to accomplish different objectives: 


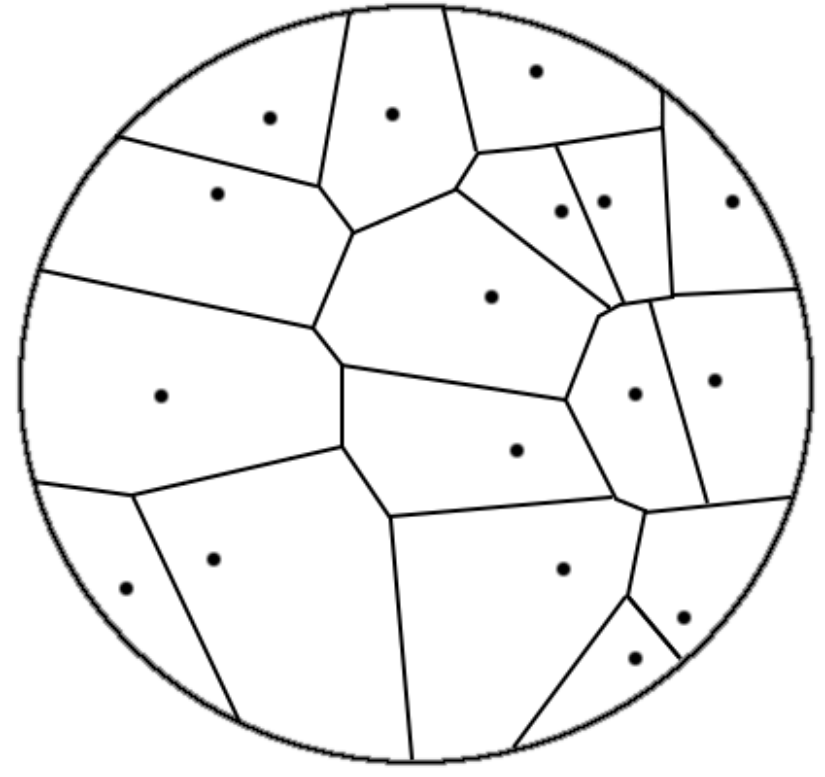

(a)

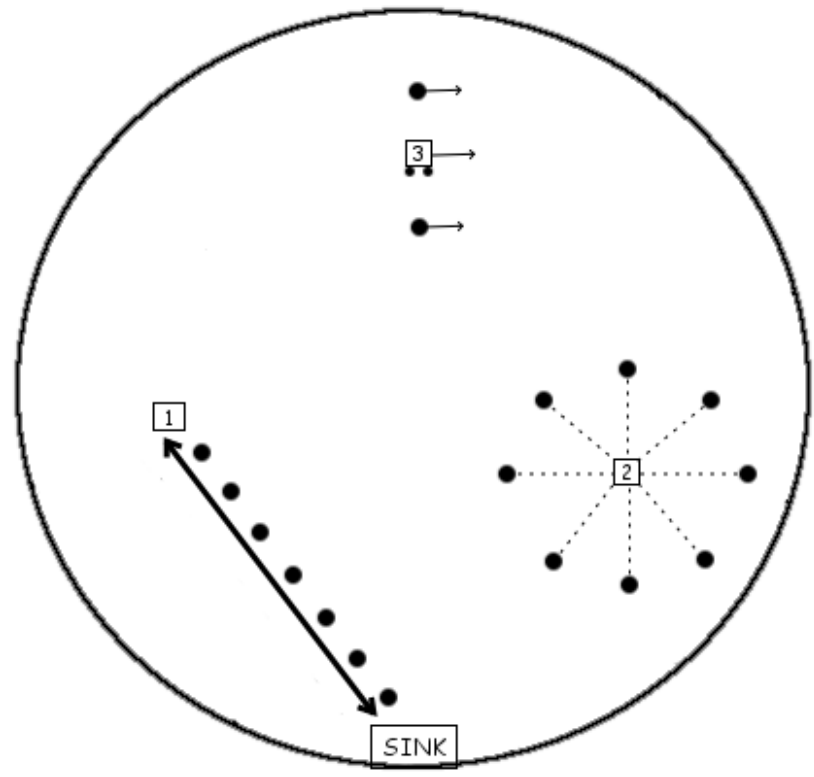

(b)

Fig. 1. Wireless sensors monitoring their Voronoi Cells (a), Wireless sensors accomplishing different tasks (b).

1) Establish a data flow between two points of the area, by moving according to a placement which guarantees the improvement of some figures of merit, such as throughput, end-to-end delay, power consumption, etc.

2) Surround, as quickly as possible, the place where an event happened, in order to gather all the required information on the phenomenon and monitor, as long as it is needed, its effects on the area under investigation.

3) Search for an intruder in the network, localize it, analyze its behavior and follow its movements.

To attain such diverse goals, nodes move according to adaptive, distributed, task-aware motion coordination and communication protocols. In this section we try to focus on the research challenges issued by the incorporation of mobility control and task coordination in the protocol stack of a wireless selforganizing sensor network. Since the number of possible objectives that nodes are called to carry out in a self-organizing network can be large, but it is still limited, in our discussion, we decide that nodes can switch among different operational modalities. Each modality corresponds to a task that nodes are trying to achieve. The behavior of the protocol stack will depend on the current modality and the modality is selected and managed by the Mobility and Task Control Plane which is transversal to the protocol stack, as shown in Fig. 2. 


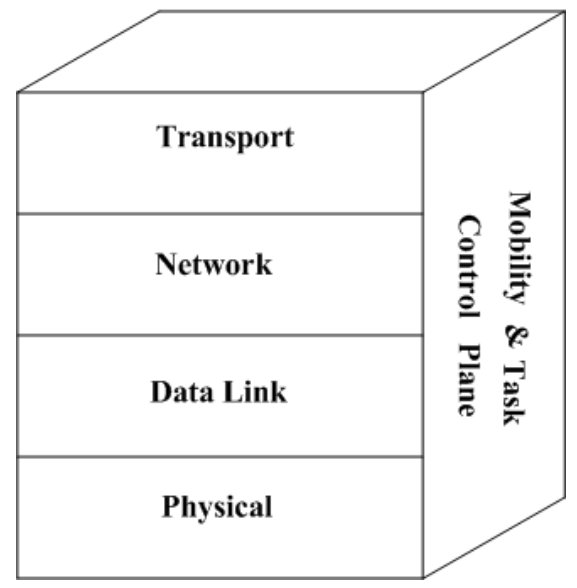

Fig. 2. Protocol stack including Mobility and Task Control Plane [21].

\section{A. Physical layer}

If the physical layer could order nodes to move, it would be, first of all, to avoid or to limit wireless channel problems. In fact, the presence of environmental obstacles and multipath effects may cause the quality of connectivity to be vastly different in different regions of the network [2]. When, instead, a task has been assigned to a node, the physical layer can highly improve the performance by determining the most suited modulation and error control coding schemes or the nodes positions which allow the least energy consumption. Lately, the physical layer community is putting a lot of effort toward the direction of exploiting cooperation among nodes through recent research works which include Virtual MIMO and physical layer relaying.

\section{B. Data Link Layer}

The continuous exchange of information between the data link layer and the mobility control can be very advantageous for the network. By knowing positions, velocities and trajectories of the nodes, the data link layer can optimize the multiplexing of data streams and build accurate collision domains. In such a dynamic environment a fine time-space scheduling policy is needed in order to avoid wasteful retransmissions and prolong network lifetime by allowing a power-saving mode of operation. Also classical problems of this layer, such as the hidden terminal or the exposed terminal, should be newly investigated because through the usage of nodes motion they could be overcome. 


\section{Network Layer}

Many procedures, typical of the network layer, are affected by the controlled mobility. Because of the dynamicity of the network, neighbour discovery becomes even more important, and it should be integrated with task discovery in order to facilitate the network organization. The network organization, in turn, is substantially modified by the movements of nodes and the assigned tasks. If sensing a phenomenon requires a clustered or a hierarchical scheme, establishing a data flow between two nodes of the network needs a linear topology. Routing has to be newly designed, because before it determined the most efficient path among deployed and static nodes, now it can look at the optimal positions where nodes have to move.

\section{Transport Layer}

Depending on the task, the transport layer issues several research challenges. In general, the end-toend paradigm does not seem to be able to keep track of dynamic multi-hop network characteristics in a responsive manner, so it should be replaced by hop-by-hop schemes, both for the rate and congestion controls. More specifically, the behavior of this layer cannot be dictated by the Round Trip Time (RTT), which can vary remarkably in different areas of the network or for different tasks. The scheduling and the fairness of data flows should take into account the availability of good quality links at programmable time instead of constant lower quality connections [19]. The measurements of the data to be transmitted, the data aggregation and the multiple flows relaying have to be reconsidered in order to meet application layer constraints.

\section{E. Cross-layer Integration}

Cross-layer integration is a "must" for controlled mobility in self-organizing networks. Different layers of the protocol stack will probably ask the nodes for diverse mobility patterns or final positions. For example, in Fig. 1 (b), when the assigned task is the establishment of a data flow between two points of the network, the network layer expects nodes to be arranged in a line in order to reduce energy consumption [1], instead, for the presence of an obstruction in the linear route, the physical layer is likely to demand a different optimal solution, even for the same objective. Likewise, the data link layer and the transport layer can have contrasting requirements in chasing an intruder - the former would try to isolate the latter, in order to have a more responsive information swap among the nodes involved in the pursuit. Generally, mobility and task control plane should constantly exchange data and requests with any layer of the stack, so as to represent the joint which makes a real cross-integration possible. 


\section{Advantages And Limitations of Controlled Mobility}

Controlled mobility has been a hot research topic of the robotics community for many years. It concerns the motion coordination of a group of robots for a common objective, typically the coverage of a geographical area. But, the number of applications where controlled mobility is beneficial is enormous, and it spreads from underwater monitoring of seismic movements to planet exploration, from environmental sensing to site surveillance and localization of intruders. The coordination requires communication, computation and control among the robots. All these aspects are covered by the vast literature of theoretical and practical results in the control theory. Instead, in the networking research world, mobility has always been seen as an issue to face more than as a facility to exploit. Only recently, has controlled mobility gained an important role also for communications matters. In the two following subsections we intend to give an overview of the possible advantages, mostly taken from recent research works, and of the limitations and the choices that a network designer should consider in order to profitably use controlled mobility.

\section{A. Advantages}

As witnessed by the recent contributions in the wireless sensor, multihop, mesh and mobile ad hoc networking, controlled mobility offers several advantages to all those kinds of wireless networks which aim to an autonomous self-organization.

The first class of parameters which can be optimized by introducing controlled mobility in wireless networks is related with power efficiency. In [1] the authors present a distributed, self-adaptive scheme of mobility control for improving power efficiency while maintaining connectivity in a wireless sensor network. More important is that they introduce mobility as a network control primitive.

Power consumption is also investigated in [2] and [3]. In [2] the authors discuss the usage of controllably mobile elements in a network infrastructure in order to reduce the energy consumption. They show that for increasing nodes densities, the presence of a mobile base station reduces the energy usage with respect to a network of static nodes. The mobility pattern of the mobile node is designed so that the path is fixed, but the speed profile followed along the path is flexible.

In [3] the authors split the nodes of a Mobile Ad-Hoc Network (MANET) in two categories: relay nodes, which are considered all mobile, and tracking nodes, which are static and used for getting information on a possible intruder of the network. They incrementally find the relays positions that minimize the total required transmission power for all the active flows in the MANET. A distributed annealing algorithm has been used for governing the motion of nodes. 
A different approach is considered in [4], where only one node is considered mobile. It can be the sink or a relay node. For the case where the sink is mobile, the upper bound on the network lifetime is analytically determined to be four times that of the static network. The authors claim that a mobile sink is not feasible, because the sink is expected to be static since it acts as gateway to a backbone network. For this reason they assume the mobile node is a relay and they construct a joint mobility and routing algorithm in order to make the network lifetime come close to the upper bound.

An even more intuitive benefit comes from the ability to control the coverage of the network, by modifying the positions of the nodes. A static network suffers from several disadvantages in covering a geographical area. First, even when the initial deployment did not leave regions uncovered, a static network can not cope with the dynamics of the environment and with the local disconnections. Second, the fixed positions of the nodes represent an easy target for a malicious attacker. Furthermore, if a specific nodes displacement is shown to be optimal for some objective, controlled mobility is the way to achieve it.

In [5] the authors design adaptive and distributed algorithms, based on Voronoi diagrams, in order to coordinate a multi-vehicle network to meet on an event point following a predefined distribution.

Butler and Rus in [6] obtain the same objective making nodes cover a given area and converge on specific points of interest in a distributed fashion. The novelty is in the absence of placements defined a priori, and in the presence of new constraints, added so as not to leave any portion of the environment uncovered. A more theoretical study is presented in [7], where the authors consider two metrics of quality of coverage $(\mathrm{QoC})$ in mobile sensor networks: the fractions of events captured and the probability that an event is captured. They provide analytical results on how these two performance metrics scale with the number of mobile sensors, their velocity patterns, and event dynamics. They also develop an algorithm for planning sensor motion such that the probability that an event is lost is bounded from above. In our opinion, an algorithm based on this work, would need each sensor to be programmed accordingly with the mobility pattern computed by a centralized unit. Two recent schemes have been introduced for maintaining connectivity through the exploitation of controlled mobility: DARA [8] e PADRA [9]. These algorithm detect possible partitions in sensor and actor networks and restore network connectivity through controlled relocation of movable nodes.

Controlled mobility can be effectively used during the network deployment phase, when an optimal placement of the nodes is too expensive or impossible due to environmental impedimenta. Reference [10] exploits the virtual force field concept for enhancing coverage. Sensors start from an initial random configuration, and, by using a combination of attractive and repulsive forces, they move to a final 
placement, where the area covered by each of them is maximized. The authors of [11] design two sets of distributed protocols, based on Voronoi diagrams, for controlling the movement of sensors to achieve target coverage. One set minimizes communications among the sensors, while the other minimizes movements. Coverage, deployment time, energy consumption and moving distances are the performance evaluation parameters used to show the effectiveness of their algorithms. In [12] authors propose two deployment algorithms to achieve sensor energy balancing and a reduced amount of energy consumption during the deployment phase. They also consider mobility of nodes and exploit it.

Controlled mobile sensors can also be used for exploration and localization, as in [13]. The cited work defines a hybrid architecture, made of a certain number of mobile actuators and a larger number of static sensors. The actuators move in the sensor-field and get information from the static sensors in order to perform site exploration, coverage repair and target localization. The algorithms which drive the actuators in their tasks are based on potential field and swarm intelligence.

Load balancing in wireless sensor networks is studied in [14]. The nodes closest to the base station are the bottleneck in the forwarding of data. A base station, which moves according to an arbitrary trajectory, continuously changes the closest nodes and solves the problem. The authors find the best mobility pattern for the base station in order to ensure an even balancement of network load on the nodes.

It is well known that, in a wireless network, the throughput degrades with the number of hops. A node, which can act as a mobile relay, would limit the number of hops and increase network performance. In literature we can find many works on data mules, whose predictable mobility is also used for improving the delivery ratio of data. In delay-tolerant networks, Message Ferrying exploits controlled mobility in order to achieve the same task of transporting data with a high delivery ratio and also where end-to-end paths do not exist between nodes. In [15] the authors propose a scheme which manages with multiple ferries and is able to meet the traffic demands while minimizing the average data delivery delay. In [16] authors propose an algorithm for joint relay node placement and node scheduling in wireless networks. They consider a system that consists of a relay node with controllable mobility and multiple nodes that communicate with each other via the relay node. The objective of their algorithm is to maximize the lowest weighted throughput among of all nodes.

A sensed phenomenon may require different rates of sampling by the sensor nodes, this leads to a nonuniform distribution of sensed data on the network and, without an accurate scheduling strategy of data collection, to a possible overflow of the buffers. In [17] the authors use mobile nodes for data gathering. First they show that the scheduling of multiple mobile elements with no data loss is a NP-complete problem, then they compare the performance of some computationally practical algorithms for single and 
multiple mobiles in terms of amount of overflow and latency in the collection of data.

In [18] the authors deform the topology of a multi-hop wireless network by moving the nodes to create new links. They show a reduction in the mean end-to-end delay of the network, even more effective than the alternative approach of increasing the capacities of the most congested network links. The algorithm is centralized and it takes as inputs: the network topology, the coordinates of the wireless nodes and the network load. Then it tries to change the network connectivity by moving the non-static network nodes in small steps. This is done such that, at each step, the network remains connected and its characteristic timescale goes down.

In Table I all the cited works are shown along with the type of wireless network under investigation and the objective of the research. In order to offer a better categorization, in the three following columns, we put the number of devices which are considered mobile, the scheme and the type of controlled mobility used in the algorithm.

Another work worth mentioning is [19]. It is not listed in Table I because the authors do not present an algorithm for the optimization of some parameters, instead they propose Morph as a new vision of sensor networking, where controlled mobility is considered as an additional design dimension of the communication protocols. They argue that, in Morph, controlled mobility can be employed for the sustainability of the network, which consists in both alleviating the lack of resources and improving the network performance.

\section{B. Limitations}

In spite of all the expected advantages that controlled mobility provides for self-organizing networks, it also poses many new questions which need to be answered before designing a protocol which envisions its employment.

First: Can the benefits introduced by controlled mobility counterbalance the expenses required by the additional hardware? A mobile node might need several extra devices for the motion, such as steering, positioning and navigation systems, in some cases it could be helpful to foresee also a remote control. Second: Does the energy budget allow the movements of one or many nodes of the network? It is necessary to define an energy model related with nodes' motion and one related with the communication needed for their coordination. For the former a simplified model is a distance proportional model $E_{m}(d)=k d+\gamma$, where $d$ is the distance to cover, $k[\mathrm{~J} / \mathrm{m}]$ takes into account the kinetic friction, while $\gamma[J]$ represents the energy necessary to overcome the static friction, both these constants depend on the environment (harsh or smooth ground, air, surface or deep water). For the latter, usually the energy required to send one bit at the distance $d$ is $E_{c}(d)=\beta d^{\alpha}$, where $\alpha$ is the exponent of the path loss 
TABLE I

RELATED WORKS

\begin{tabular}{|c|c|c|c|c|c|}
\hline Algorithm & Type of Network & Objective & $\begin{array}{c}\text { \# of Mobile } \\
\text { Devices }\end{array}$ & Scheme & $\begin{array}{c}\text { Type of Controlled } \\
\text { Mobility }\end{array}$ \\
\hline $\begin{array}{l}\text { Mobility } \\
\text { Control [1] }\end{array}$ & Multihop WN & $\begin{array}{c}\text { Connectivity } \\
\text { Energy Consumption }\end{array}$ & All & Distributed & Adaptive \\
\hline $\begin{array}{c}\text { Adaptive } \\
\text { Motion [2] }\end{array}$ & WSN & Energy Consumption & Sink & - & Programmed \\
\hline $\begin{array}{c}\text { Distributed } \\
\text { Annealing [3] }\end{array}$ & MANET & Energy Consumption & All & Distributed & Adaptive \\
\hline ARALN [4] & WSN & Network Lifetime & Single node & - & Programmed \\
\hline $\begin{array}{c}\text { Coverage } \\
\text { Behavior [5] }\end{array}$ & WSN & Coverage & All & Distributed & Adaptive \\
\hline $\begin{array}{l}\text { Local } \\
\text { Voronoi [6] }\end{array}$ & WSN & Coverage & All & Distributed & Adaptive \\
\hline BELP [7] & WSN & Quality of Coverage & To be computed & Centralized & Programmed \\
\hline DARA [8] & WSAN & Connectivity & Some & Distributed & Adaptive \\
\hline PADRA [9] & WSAN & Connectivity & Some & Distributed & Adaptive \\
\hline VFA [10] & WSN & Deployment & All & Distributed & Adaptive \\
\hline $\begin{array}{c}\text { VEC, VOR, } \\
\text { Minimax [11] }\end{array}$ & WSN & Deployment & All & Distributed & Adaptive \\
\hline $\begin{array}{c}\text { Deployment } \\
\text { Algorithms [12] }\end{array}$ & WSN & Deployment & All & Distributed & Adaptive \\
\hline TARANTULAS [13] & WSAN & Localization & Some & Distributed & Adaptive \\
\hline $\begin{array}{c}\text { Joint Mobility } \\
\text { and Routing [14] }\end{array}$ & WSN & Load Balancing & Sink & - & Programmed \\
\hline MURA [15] & DTN & Delivery Ratio & Some & Centralized & Programmed \\
\hline $\begin{array}{c}\text { Placement } \\
\text { \&Scheduling [16] }\end{array}$ & Multihop WN & Throughput & Single Node & Centralized & Adaptive \\
\hline MES [17] & WSN & No Data Loss & Some & Distributed & Adaptive \\
\hline $\mathrm{CD}[18]$ & Multihop WN & End-to-end delay & All & Centralized & Adaptive \\
\hline
\end{tabular}


$(2 \leq \alpha \leq 6)$ depending on the environment and $\beta$ is a constant $\left[\mathrm{J} /\left(\right.\right.$ bits $\left.\left.\cdot \mathrm{m}^{\alpha}\right)\right]$.

Third: If it is needed by the application, can the controlled mobility guarantee the connectivity at the intermediate steps in the process of new displacement of the nodes? Applications with real-time, delaysensitive, continuous flow of data constraints do not tolerate node failures or local disconnections. For these kinds of applications, mobility should only operate when connectivity is not compromised by nodes movement.

Fourth: What number of nodes should be mobile? How is the mobility pattern chosen? Considering that only the sink is mobile brings evident advantages [2], [4], [14] but, usually, that specific node is in charge of bridging self-organizing and backbone networks. However, a reduced number of mobile nodes represents a viable answer to the power consumption problem and an effective solution for some parameter optimization [13], [15], [17]. A tradeoff between the number of mobile nodes and the energy consumption has to be determined depending on the application demands. This compromise has to take into consideration also the determination of a mobility pattern that, if it is not selected appropriately, can cause a larger waste of resources.

Fifth: What happens if network dynamics are too fast with respect to nodes convergence through mobility? And if, for some reason, nodes can not reach the final, expected configuration, may an intermediate displacement be disadvantageous? A wrong placement of the nodes can transform the positive effects that controlled mobility was meant to introduce into a worse overall performance of the network. As seen in Table I, many schemes give the nodes the ability to move adaptatively to a task to perform or an event to track. This behavior is a primary concern in self-organizing networks design, since it gives the system the ability to react, in a distributed or a centralized fashion, to the changes in the applications demands and in the time-space constraints.

\section{Case Study: Mobility as a Network Control Primitive}

In this section we analyze the work in [20] as a case study. In [20], the authors developed a mathematical model for determining the best placement of nodes by taking into consideration the energy of each node. The maximization of the shortest node's lifetime is achieved. Fig.4 (a) shows the network under examination. It consists of a certain number of nodes, which appear randomly distributed in the field. In this field, the source $\mathrm{S}$ activates a data flow to the destination $\mathrm{D}$, these terminal nodes are the diamonds. The straight line represents the direct path between source and destination. The filled circles are the nodes chosen by the routing algorithm in their original positions. It is worth to note that in this work we are not interested on how nodes achieve optimal positions. In this scenario, we assume there exists a motion scheme such as [5] that allow nodes to move from their original positions to the optimal positions. 


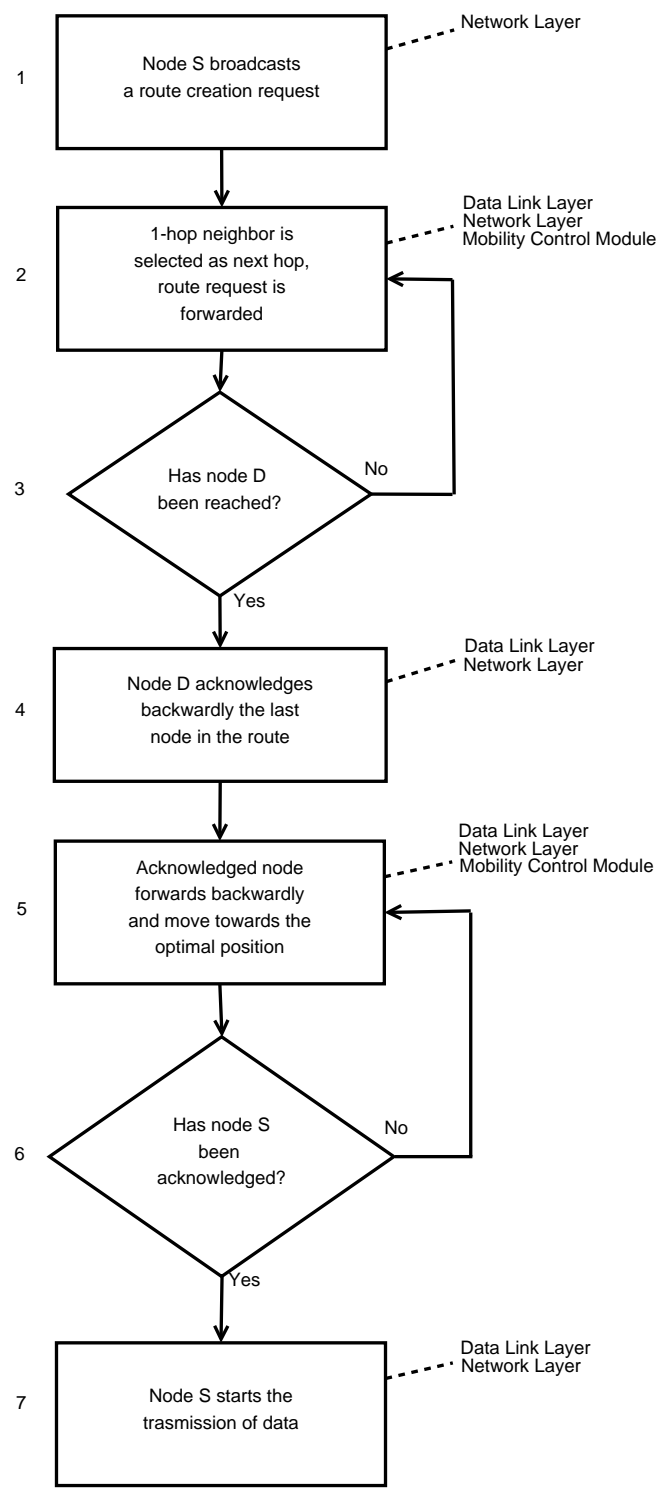

Fig. 3. Flowchart for the case study algorithm.

In order to do this, we introduce an algorithm which implements the cited scheme and makes the following assumptions:

1) Source does not have a route to the destination yet.

2) The data link layer is provided with a scheduler which avoids collisions among concurrent transmitting nodes.

3) Nodes know their positions.

In the flow chart in Fig. 3, we describe the steps of the algorithm which starts when the source requests 
to activate a data flow to the destination. Next to each step of the algorithm we indicated the layer or module involved. In particular, in Step 2, the choice of the next-hop among the neighboring nodes that received the request is executed depending on criteria based, for example, on their residual energy and current distance from the straight path. The acknowledgement sent backwardly in Step 4-5 has the twofold purpose to establish the end-to-end connection and to inform nodes belonging to the route that they can move.

The effects of the mobility are illustrated in Fig.4 (b) where nodes moved from their original positions, which are crossed in the figure, to the positions computed through the asynchronous algorithm of [20], along the segment between source and destination. These positions will allow nodes to consume the minimum amount of energy for their communications.

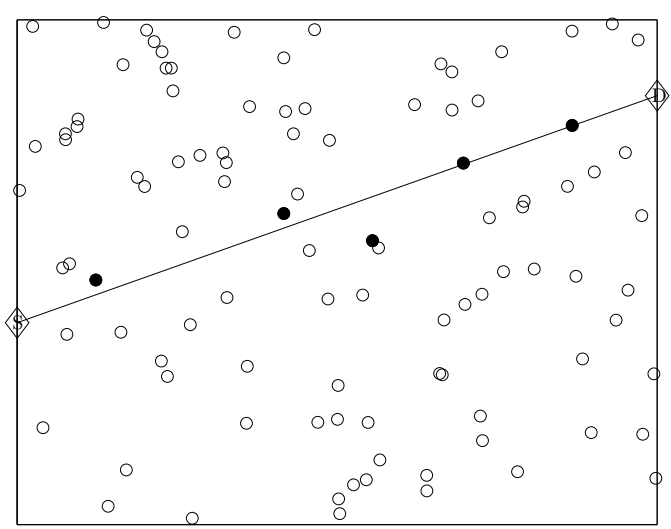

(a)

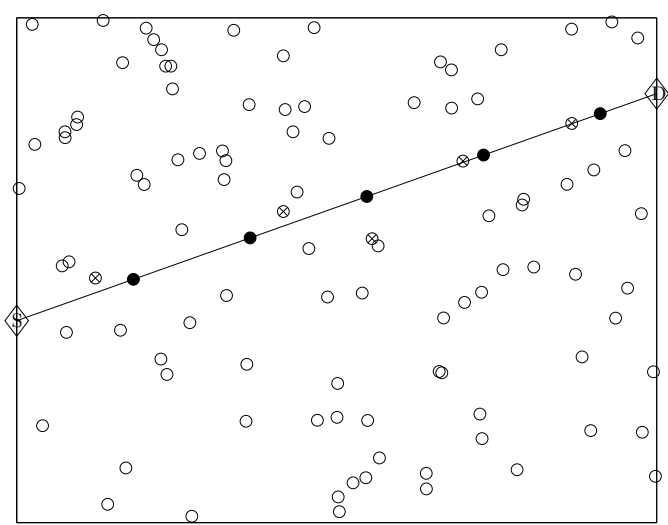

(b)

Fig. 4. Initial positions of nodes chosen by the routing (a), Nodes moved to the evenly spaced positions on the straight route between source and destinations (b).

\section{CONCLUSiON}

In this paper we focused on the controlled mobility in self-organizing wireless networks. If mobility is considered as a network control primitive it creates a series of new interactions with the protocol stack, which we illustrated layer by layer for a wireless sensor network. We illustrated the advantages of controlled mobility and showed some of the possible limitations in using it as a new design dimension. Finally, we exemplified further through a case study a possible scheme to use controlled mobility and showed the dependencies of each step of the scheme with the protocol stack. 


\section{REFERENCES}

[1] D.K. Goldenberg, J. Lin, A.S. Morse, B.E. Rosen and Y.R. Yang, "Towards Mobility as a Network Control Primitive," in Proceedings of ACM Mobihoc, Page(s):163-174, 2004.

[2] A.A. Somasundara, A. Kansal, D.D. Jea, D. Estrin and M.B. Srivastava, "Controllably mobile infrastructure for low energy embedded networks," in IEEE Transactions on Mobile Computing, Volume:5, Issue:8, Page(s):958-973, 2006.

[3] R. Rao and G. Kesidis, "Purposeful Mobility for Relaying and Surveillance in Mobile Ad Hoc Sensor Networks," in IEEE Transactions on Mobile Computing, Volume:3, Issue:3, Page(s):225-232, 2004.

[4] W. Wang, V. Srinivasan and K.C. Chua, "Using Mobile Relays to Prolong the Lifetime of Wireless Sensor Networks," in Proceedings of ACM MOBICOM, Page(s):270-283, 2005.

[5] J. Cortes, S. Martinez, T. Karatas and F. Bullo, "Coverage Control for Mobile Sensing Networks," in IEEE Transactions on Robotics and Automation, Volume:20, Issue:2, Page(s):243-255, 2004.

[6] Z. Butler and D. Rus, "Controlling Mobile Sensors for Monitoring Events with Coverage Constraints," in Proceedings of IEEE ICRA, Volume:2, Page(s):1568-1573, 2004.

[7] N. Bisnik, A. Abouzeid and V. Isler, "Stochastic Event Capture Using Mobile Sensors Subject to a Quality Metric," in IEEE Transactions on Robotics, Volume:23, Issue:4, Page(s):676-692, 2007.

[8] A. A. Abbasi, M. Younis and K. Akkaya, "Movement-Assisted Connectivity Restoration in Wireless Sensor and Actor Networks," in IEEE Transactions on Parallel and Distributed Systems, Volume 20 Issue 9, September 2009.

[9] K. Akkaya, F. Senel, A. Thimmapuram and S. Uludag, "Distributed Recovery from Network Partitioning in Movable Sensor/Actor Networks via Controlled Mobility," in IEEE Transactions on Computers, vol. 59, no. 2, pp. 258-271, Feb. 2010.

[10] Y. Zou and K. Chakrabarty, "Sensor deployment and target localization in distributed sensor networks," in ACM Transactions on Embedded Computing Systems, Volume:3, Page(s):61-91, 2004.

[11] G. Wang, G. Cao and T.F. La Porta, "Movement-Assisted Sensor Deployment," in IEEE Transactions on Mobile Computing, Volume:5, Issue:6, Page(s):640-652, 2006.

[12] S. Abdel-Mageid and R.A. Ramadan, "Efficient deployment algorithms for mobile sensor networks," in IEEE International Conference of Autonomous and Intelligent Systems (AIS), June 2010.

[13] W.K.G. Seah, K.Z. Liu, M.H. Ang Jr., J.G. Lim, and S.V. Rao, "TARANTULAS: Mobility-enhanced Wireless SensorActuator Networks," in Proceedings of IEEE Sensor Networks, Ubiquitous, and Trustworthy Computing, Volume:1, Page(s):548-551, 2006.

[14] J. Luo and J.P. Hubaux, "Joint Mobility and Routing for Lifetime Elongation in Wireless Sensor Networks," in Proceeding of IEEE INFOCOM, Volume:3, Page(s):1735-1746, 2005.

[15] W. Zhao, M. Ammar and E. Zegura, "Controlling the Mobility of Multiple Data Transport Ferries in a Delay-Tolerant Network," in Proceedings of IEEE INFOCOM, Volume:2, Page(s):1407-1418, 2005.

[16] H.-T. Roh and J.-W. Lee, "Joint relay node placement and node scheduling in wireless networks with a relay node with controllable mobility," Wireless Communications and Mobile Computing, n/a. doi: 10.1002/wcm.1007.

[17] A.A. Somasundara, A. Ramamoorthy and M.B. Srivastava, "Mobile Element Scheduling with Dynamic Deadlines," in IEEE Transactions on Mobile Computing, Volume:6, Issue:4, Page(s):395-410, 2007.

[18] A. Basu, B. Boshes, S. Mukherjee and S. Ramanathan, "Network Deformation: Traffic-Aware Algorithms for Dynamically Reducing End-to-end Delay in Multi-hop Wireless Networks," in Proceedings of ACM MobiCom, Page(s):100-113, 2004.

[19] A. Kansal, M. Rahimi, D. Estrin, W.J. Kaiser, G.J. Pottie and M.B. Srivastava, "Controlled Mobility for Sustainable Wireless Sensor Networks," in Proceedings of IEEE SECON, Page(s):1-6, 2004. 
[20] E. Natalizio, V. Loscrí and E. Viterbo, “Optimal Placement of Wireless Nodes for Maximizing Path Lifetime,” in IEEE Communication Letters, Vol. 12, Issue 5 Page(s):362-364, 2008.

[21] I. F. Akyildiz, W. Su, Y. Sankarasubramaniam and E. Cayirci, "Wireless Sensor Networks: A Survey," in Elsevier Computer Networks, Volume:38, Issue:2, Page(s):393-422, 2002.

\section{SHORT BIOGRAPHIES}

Enrico Natalizio obtained his master's degree magna cum laude and his $\mathrm{Ph} . \mathrm{D}$ in Computer Engineering at the Universitá della Calabria in 2000 and 2005 respectively. During his last year of doctoral studies he joined the BWN (Broadband Wireless Networking) Lab at Georgia Tech in Atlanta, GA, USA under the supervision of Prof. Akyildiz. Since December 2005 he has been a research fellow and a contract professor at the Department of Electronics, Computer and System Sciences, Universitá della Calabria, Italy. Since October 2010, he joined POPS team at INRIA-Nord Europe as a postdoc fellow for the ARC MISSION project.

Valeria Loscrí received the Laurea Degree in Computer Science and the Ph.D. in Information Science, from the University of Calabria (UNICAL), Italy, in 2003 and 2007 respectively. Currently she is with the Department of Electronics, Computer Science and Systems (D.E.I.S.) of the University of Calabria as Research Fellowship with the Telecommunication group. Her current research interests include Mobile and Wireless Networks and Performance Evaluation of Communication Networks. From January 2006 to July 2006 she has been visiting researcher in the Rice Network Group at the Rice University of Houston (TX) and her supervisor has been Prof. Edward Knightly. 